\title{
Causes of extremely fast CMEs
}

\author{
Joan Feynman and Alexander Ruzmaikin \\ Jet Propulsion Laboratory, California Institute of Technology, Pasadena, CA 91109, USA \\ email: Joan.Feynman@jpl.nasa.gov
}

\begin{abstract}
We study CMEs observed by LASCO to have plane of the sky velocities exceeding $1500 \mathrm{~km} / \mathrm{sec}$. We find that these extremely fast CMEs are typically associated with flares accompanied by erupting prominences. Our results are consistent with a single CME initiation process that consists of three stages. The initial stage is brought about by the emergence of new magnetic flux, which interacts with the pre-existing magnetic configuration and results in a slow rise of the magnetic structure. The second stage is a fast reconnection phase with flaring, filament eruption and a sudden increase of the rise velocity of the magnetic structure (CME). The third stage consists of propagation in the corona. We discuss the sources of these CMEs and the need for improved understanding of the first and third stages.
\end{abstract}

Keywords. Sun:coronal mass ejections (CMEs), Sun: prominences, Sun: flares, Sun: magnetic fields

\section{Introduction}

The distribution of the plane of the sky velocities of CMEs observed by SOHO is shown in Figure 1 (Yurchyshyn et al., 2005). This distribution of $4315 \mathrm{CMEs}$ shows few CMEs with velocities greater than $1500 \mathrm{~km} / \mathrm{sec}$ and none with velocities exceeding $2000 \mathrm{~km} / \mathrm{sec}$. However these are the CMEs that cause the most important solar energetic particle events and the most intense geomagnetic storms. Here we report on our studies of two populations: 23 CMEs with $v>1500 \mathrm{~km} / \mathrm{sec}$ during the years 1989-2001 (Feynman \& Ruzmaikin 2004), and 22 CMEs with $v>2000 \mathrm{~km} / \mathrm{sec}$ during the years 2001 to 2005 . All of these CMEs were clearly associated with flares on the visible Sun. Normal velocity CMEs are categorized as being associated with either a flare or a disappearing filament whereas extreme velocity CMEs are typically associated with both a flare and a filament eruption. Normal velocity CMEs associated with filament disappearances typically show constant acceleration in the corona whereas those associated with flares usually show a constant speed or decreasing speed (Sheeley et al. 1999;Andrews\& Howard 2001; Moon et al. 2002). Extremely fast CMEs $(v>1500 \mathrm{~km} / \mathrm{sec})$ are as likely to show constant acceleration as constant or decreasing speed (Feynman \& Ruzmaikin 2004).

\section{Extremely fast CMEs}

Figure 1b shows the distribution in time of CMEs with $v>2000 \mathrm{~km} / \mathrm{sec}$ observed during 2001-2005. Notice that they are not distributed at random. They tend to occur in sets. For example, four superfast CMEs occurred in April 2001, seven occurred during the Halloween events of October/November 2003, and four took place in January 2005. Each of these sets is associated with long lived cluster of activity. Figure 2 exemplifies the relation of a super-event to emerging flux in agreement with the suggestions of Feynman \& Martin (1995) and Feynman (1997). Feynman \& Ruzmaikin (2004) stressed the importance of long-lived clusters of activity (Gaizauskas et al. 1983) as sources of the 

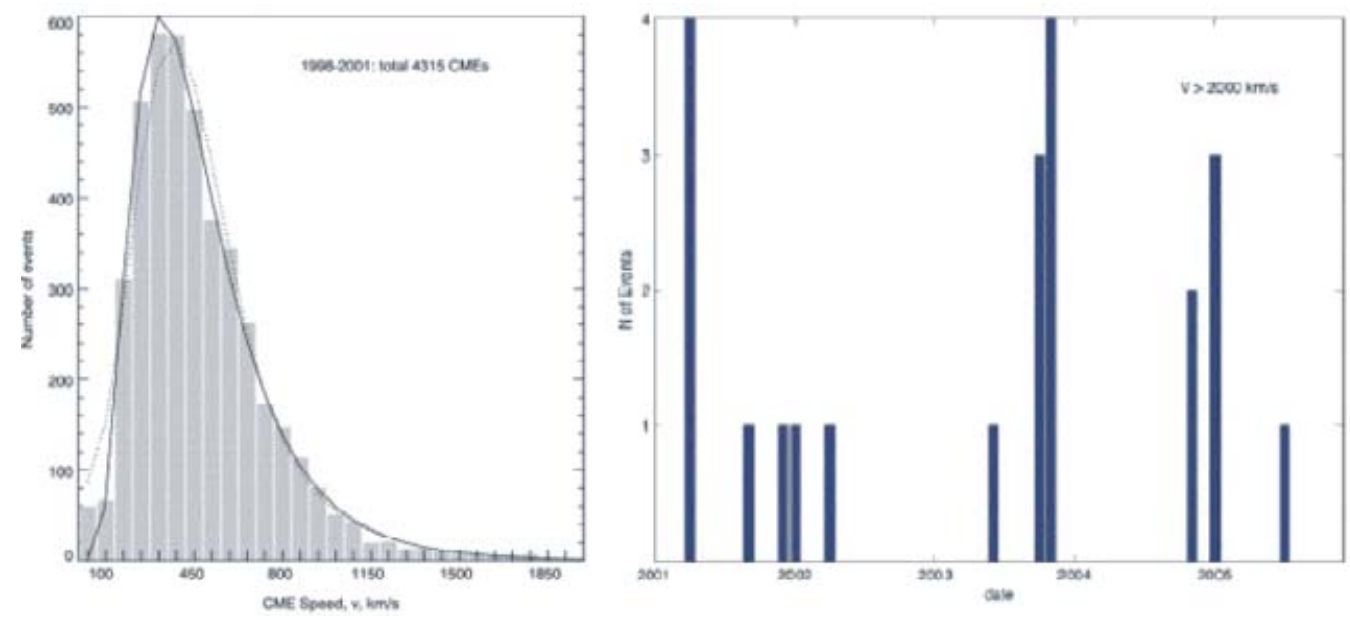

Figure 1. (a) Distribution of the CME speeds (Yurchyshyn et al., 2005). Speeds exceeding 1500 $\mathrm{km} / \mathrm{s}$ are very rare, exceeding $2000 \mathrm{~km} / \mathrm{s}$ are off scale. (b) CMEs with $v>2000 \mathrm{~km} / \mathrm{sec}$ observed in 2001-2005.

Table 1. Three extremely fast CMEs

\begin{tabular}{lcccc}
\hline Date & $v \mathrm{~km} / \mathrm{s}$ & Kin. Energy (egr) & X-ray Flare & Filament \\
\hline 12 Sep 2000 & 1550 & $2.6 \mathrm{e}+32$ & $\mathrm{M} 1$ & eruption \\
21 Apr 2002 & 2408 & - & M1.5 & eruption \\
28 Oct 2003 & 2459 & $1.2 \mathrm{e}+33$ & $\mathrm{X} 17.2$ & eruption \\
\hline
\end{tabular}

fast CMEs. The left panel shows a full disk solar magnetogram from Kitt Peak, October 26, 2003. The large southern activity region near central meridian (10486) was also present on the previous rotation and the next rotation, so it qualifies as a long-lived cluster of activity. A comparison with the October 28 magnetogram (right panel) shows newly emerging flux (region 10488) in the North at almost the same solar longitude. This new region persisted for two more rotations and therefore was also a long-lived active region. The change of relative helicity in the region 10488 during this flux emergence has been estimated by Liu \& Zhang (2006) who report an increase estimated to be about that which left the Sun in the two CMEs they associate with region 10488. However, magnetic connections between region 10488 and 10486 could be seen to form as coronal loops 14 hours after the emergence began. During the next 31 hours, frequently changing connecting loops were present. These connections introduce some uncertainty into the Liu \& Zhang estimate of the helicity change induced by the 10486 emerging flux because the method used requires that the flux of the studied region be isolated (i.e. that the surface of a volume can be defined enclosing the region such that no field lines cross that surface above the photosphere.) The combined activity center 10488 plus 10486 was associated with the seven CMEs with velocity exceeding $2000 \mathrm{~km} / \mathrm{sec}$ (Figure 1b); surely an extremely rare event. Table 1 shows some selected properties of three extremely fast CMEs. Each event is associated with a long-lived cluster of activity and is accompanied by a flare and filament disappearance. Surprisingly only one of the flares is class X. The other two are more moderate class $M$ flares, suggesting there is not a one-to-one relationship between flare class and CME speed. All three CMEs are extremely large and bright and when the kinetic energy could be determined it was very high. 


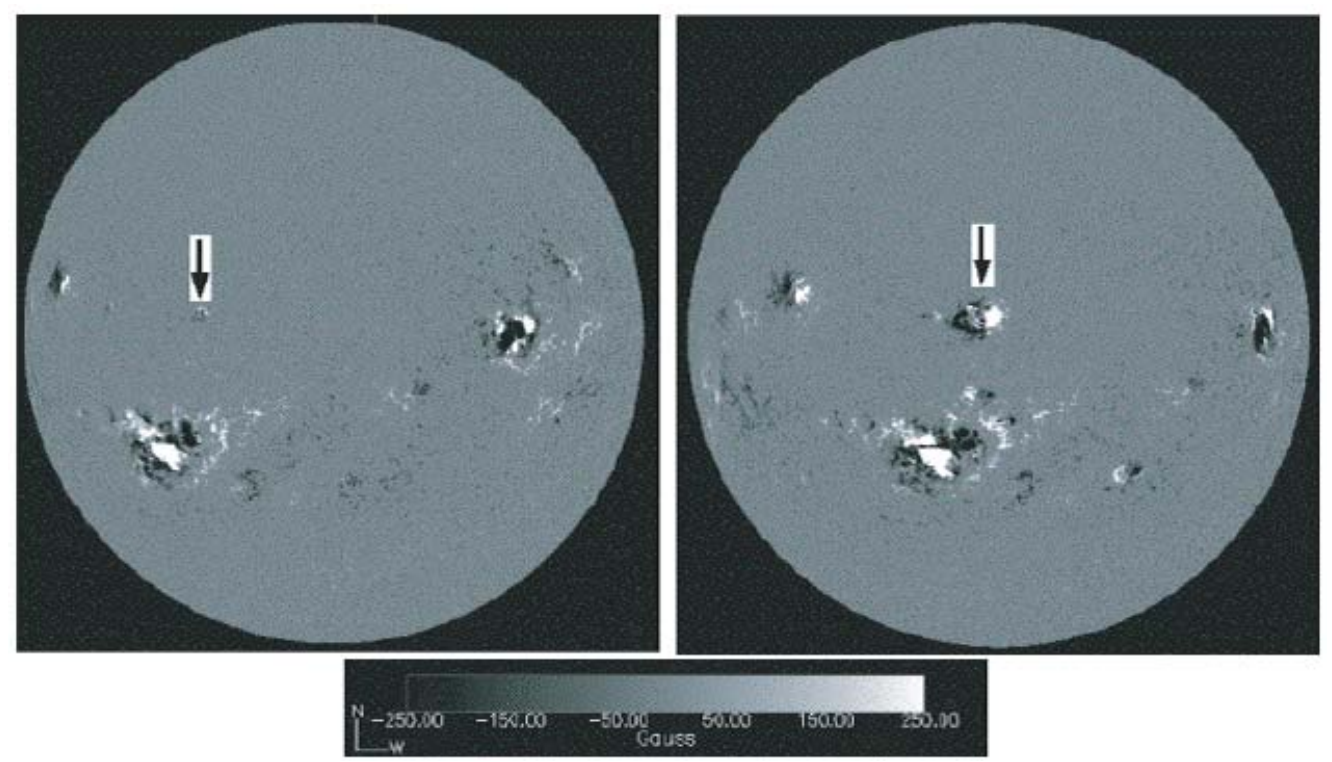

Figure 2. Top panel, Oct 26, 2003. Bottom panel Oct 28, 2003.

\section{Disscussion}

These three events are apparently examples of the three stage process of producing extremely fast CMEs suggested earlier (Zhang et al 2001; Gallagher, Lawrence \& Dennis 2003; Feynman \& Ruzmaikin 2004). In the first stage new flux emerges and interacts with existing strong magnetic fields of a cluster of activity, changing the field configurations and the relative helicity. After some hours or days the equilibrium of the cluster of activity is destroyed and the filament begins to rise slowly. The second stage begins some hours later when a second destabilization occurs and the filament rises rapidly, its helical structure becomes evident, flares occur in various spectral lines and the CME rises through the corona. Both the initial slow rise and the subsequent fast acceleration are typical features of filament eruptions (Rompolt 1990; Vršnak, Ruždjak \& Rompolt 1991). Although the first slow rise phase is observed routinely in filament eruptions and therefore is part of the process of producing fast CMEs. It is not usually included in the CME initiation modeling. The second (fast rise) stage is believed to be due to magnetic reconnection. The third stage is the propagation of the $\mathrm{CME}$ in the corona where its velocity is measured. The causes of the observed variations in the form of the velocity profile (accelerating or decelerating profiles) are not well understood, although Feynman \& Ruzmaikin (2004) have suggested that the differences are due to the altitude in the corona at which the second stage begins. These studies indicate that both the first and third stages require further empirical and modeling studies.

\section{Acknowledgements}

The CME data are taken from the SOHO LASCO CME catalog generated and maintained by NASA and The Catholic University of America in cooperation with the Naval Research Laboratory. SOHO is a project of international cooperation between ESA and NASA. Data on filaments are from the National Geophysical Data Center and the Space Environment Center supported by the National Oceanic and Atmospheric Administration (NOAA), US Department of Commerce. This work was supported by the Jet Propulsion 
Laboratory of the California Institute of technology, under a contract with the National Aeronautics and Space Administration.

\section{References}

Andrews, M. D. \& Howard, R. A. 2001, Space Sci. Rev., 95, 147

Delannee, C., J.- P. Delaboundiniere, \& Lamy, P. 2000, Astron \& Atrophys., 355, 725

Feynman, J. 1997, in: N. Crooker, J.-A. Joselyn \& J. Feynman (eds.), Coronal Mass Ejections, Geophysical Monograph 99, American Geophysical Union, p. 49

Feynman, J. \& Martin, S. F. 1995, J. Geophys. Res., 100, 3355

Feynman, J. \& Ruzmaikin, A. 2004, Solar Phys., 219, 301

Gaizauskas, V., K. L. Harvey, J. W. Harvey \& C. Zwaan, ApJ, 265, 1056

Gallagher, P. T., Lawrence, G. R., \& Dennis, B. R. 2003, Astrophys. J., 588, L53

Liu, J. \& Zhang, H. 2006, Solar Phys., 2234, 21

Moon, Y.-J., Choe, G. S., Wang, H., Park, Y.D., Gopalswamy, N., Yang, Guo, \& Yashiro, S. 2002, ApJ, 581, 694

Rompolt, B. 1990, Hvar Obs. Bull., 14, 37

Sheeley, N.R., Walters, J. H., Wang, Y.-M., \& Howard, R. A. 1999, J. Geophys. Res., 104, 24,739

Vršnak, B., Ruždjak, V. \& Rompolt 1991, Solar Phys., 136,151

Wang, H., Qui, J., Jing, J., \& Zhang, H. 2003, ApJ, 593, 564

Wang, Y.-M., and Sheeley, N.R., Jr. 1999, ApJ, 510, L157

Yurchyshyn, V., S. Yashiro, V. Abramenko, H. Wang \& N. Gopalswamy, ApJ, 619, 599

Zhang, J., Dere, K. P., Howard, R. A., Kundu, M. R., \& White, S. M. 2001, ApJ, 559, 452 